\title{
Structure Determination of Glycinocins A to D, Further Evidence for
}

\author{
the Cyclic Structure of the Amphomycin Antibiotics
}

\author{
FANGMING KonG* and GuY T. CARTER \\ Natural Products Chemistry, Wyeth Research, \\ 401 N. Middletown Road, Pearl River, NY 10965, USA
}

(Received for publication March 31, 2003)

\begin{abstract}
Four novel cyclolipopeptides, glycinocins A to D, were isolated from the fermentation broth of an unidentified terrestrial Actinomycete species. These compounds were separated and purified from the fermentation broth by 1-butanol extraction, followed by repeated reversedphase HPLC. Their structures were elucidated by spectroscopic and chemical degradation studies. The absolute configuration of the amino acid residues was determined using Marfey's methodology. The glycinocin antibiotics are structurally related to amphomycin that was originally reported as a linear lipopeptide with $C$-terminal diketopiperazine moiety. Our degradation study of the glycinocin antibiotics also yielded diketopiperazine-containing fragments, but these have been shown to be hydrolytic by-products generated by condensation of the pipecolinic acid and diamino propionic acid residues.
\end{abstract}

In the course of our ongoing search for antibacterial leads from microorganisms ${ }^{1)}$, an unidentified terrestrial Actinomycete, designated AW998, was found to produce new lipopeptide antibiotics. Lipopeptides constitute a broad and well-known family of antibiotics ${ }^{2}$. In this paper, we report the isolation and structure determination of the glycinocin antibiotics (Figure 1), and provide additional evidence that this antibiotic family, typified by amphomycin, does contain a cyclic peptide core. Taxonomy and fermentation of the producing organism as well as the biological activity will be reported elsewhere.

\section{Results and Discussion}

\section{Isolation}

The glycinocin antibiotics were mainly present in the fermentation broth, although a small amount of material was also associated with the cell mass. The metabolites were recovered by extraction of the filtrate with $n$-butanol and by methanol treatment of the mycelial mass of the AW998 culture. The antibiotic activity was concentrated in the $n-\mathrm{BuOH}$ layer based on assay against Staphylococcus aureus. Both $n-\mathrm{BuOH}$ and $\mathrm{MeOH}$ extracts were fractionated by reversed phase HPLC and the peaks were detected by UV absorbance at $215 \mathrm{~nm}$. The antibiotics were eluted in the following sequence: glycinocins C (3), D (4), A (1), and B (2). All compounds were finally obtained as white amorphous powders after lyophilization.

\section{Structure Determination}

Glycinocin A (1) was obtained as an optically active compound with a UV spectrum showing only end absorption. High resolution FT-ICR mass spectrometry yielded a molecular weight of 1246.6453, appropriate for a molecular formula of $\mathrm{C}_{57} \mathrm{H}_{90} \mathrm{~N}_{12} \mathrm{O}_{19}$. Examination of the NMR data acquired on 1 quickly identified that the molecule was a lipopeptide.

Identification of the $\alpha, \beta$-unsaturated fatty acid residue present in the molecule was straightforward. The proton NMR spectrum of 1 showed two olefinic proton resonances at $\delta 5.93(\mathrm{H}-2)$ and $\delta 6.62(\mathrm{H}-3)$ with a coupling constant of $15.6 \mathrm{~Hz}$, indicating an $E$-configuration. In the HMQC spectrum, H-3 was attached to a deshielded olefinic carbon resonance at $\delta 143.3(\mathrm{C}-3)$ and $\mathrm{H}-2$ was correlated to a

\footnotetext{
* Corresponding author: kongf@wyeth.com
} 
Fig. 1. Structures of glycinocins A to D antibiotics $(\mathbf{1} \sim \mathbf{4})$.

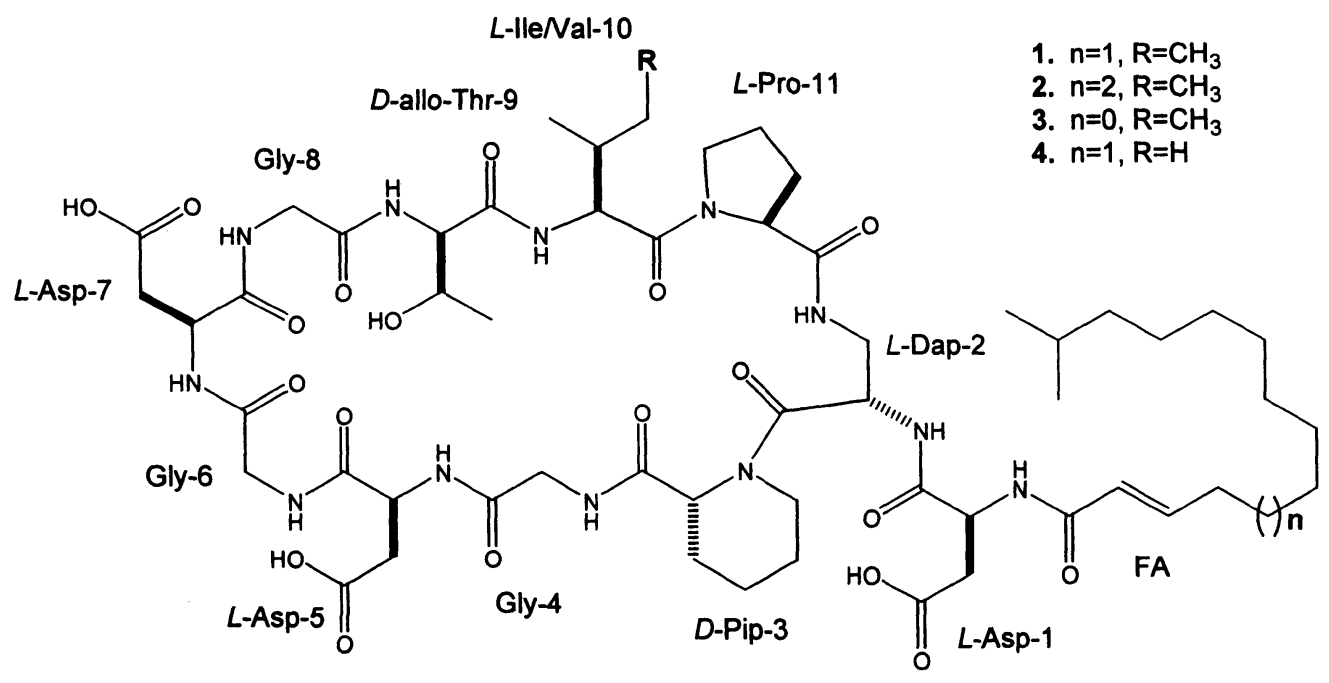

shielded carbon signal at $\delta 124.1$ (C-2), typical chemical shifts for a polarized double bond. HMBC correlations observed from both $\mathrm{H}-2$ and $\mathrm{H}-3$ to the most shielded carbonyl resonance at $\delta 165.1$, appropriate for a conjugated carbonyl, confirmed the existence of an $\alpha, \beta$-unsaturated carbonyl system. A methyl doublet at $\delta 0.8$, integrating for six protons, was evidence that the fatty acid chain terminated in an isopropyl group. Further evidence for the fatty acid substructure came from chemical degradation of the natural product $\mathbf{1}$, which yielded a hydrophobic fragment IV. Detailed analysis of the COSY, HMQC, and HMBC data acquired on IV established its identity as $N$-(2isopentadecenoyl) aspartic acid, that is, the fatty acyl amide of aspartic acid. The length of the fatty chain was revealed by the MS data (see Experimental).

The peptide nature of $\mathbf{1}$ was apparent from a series of exchangeable amide protons between $\delta 7.5$ and $\delta 8.5$ that were coupled to $\alpha$-methine protons or $\alpha$-methylene protons between $\delta 3.0$ and 4.4. In the ${ }^{13} \mathrm{C}$ NMR spectrum, a cluster of downfield carbon resonances observed between $\delta 168$ and $\delta 172$ was appropriate for amide or carboxylic acid carbonyls. Extensive NMR analyses of compound 1, including data from COSY, TOCSY, HMQC, HMBC, and HSQC-TOCSY experiments, suggested the presence of aspartic acid, glycine, threonine, isoleucine, proline, and 2,3-diaminopropionic acid (Dap) residues (Table 1). However, due to the broadened and poorly resolved signals in the ${ }^{1} \mathrm{H}$ and ${ }^{13} \mathrm{C}$ NMR spectra, it was not feasible to fully assign the structure by NMR. For example, it was extremely difficult to identify the pipecolic acid residue as a result of the severely overlapping methylene signals.

\section{Partial Acid Hydrolysis}

Glycinocin A (1) was subjected to hydrolytic conditions in $3 \mathrm{~N}$ hydrochloric acid solution $\left(\mathrm{MeOH}-\mathrm{H}_{2} \mathrm{O}=1: 1\right)$ heated at $80^{\circ} \mathrm{C}$. The reaction mixture was then separated by preparative HPLC using a linear gradient from $50 \%$ to $80 \%$ $\mathrm{MeCN}-0.1 \%$ TFA buffer to give a series of products eluting in order: I, II, III, and IV. The most polar fraction I was further purified using the same HPLC column with a weaker gradient of $5 \%$ to $25 \% \mathrm{MeCN}-0.1 \%$ TFA buffer to yield the polar fragments: Ia, Ib, Ic, and Id (Figure 2). The front peak that contained the hydrolyzed glycine and aspartic acid residues was not characterized.

Spectroscopic analyses of the chemical degradation products led to identification of all the fragments. The polar fragments were identified to be a diketopiperazine consisting of Dap and Pip (Ia), a tetrapeptide Thr-Ile-ProDap (Ib), a tripeptide Thr-Ile-Pro (Ic), and a pentapeptide with the Dap-Pip diketopiperazine as the $C$-terminal (Id). All the non-polar fragments were found to contain the fatty acyl moiety, which was consistent with their lower reversed phase HPLC mobility. They were: Thr-Ile-Pro-Dap-Asp-FA (II), a succinimido derivative (III), and Asp-FA (IV). The structures of fragments Ia, Ic, and Ib secured the sequence of the larger fragment Id. The diketopiperazine terminus of Id appeared to support the previously reported linear 
Table 1. ${ }^{1} \mathrm{H}$ and ${ }^{13} \mathrm{C}$ NMR assignments for glycinocin A (1) in DMSO- $d_{6}$.

\begin{tabular}{|l|l|l|l|l|}
\hline Residue & $\begin{array}{l}{ }^{13} \mathrm{C} / \\
100 \mathrm{MHz}\end{array}$ & $\begin{array}{l}\mathrm{H} / 500 \mathrm{MHz} \\
\text { Mult }(\mathrm{J}, \mathrm{Hz})\end{array}$ & $\begin{array}{l}\text { Key-TOCSY } \\
\text { correlations }\end{array}$ & $\begin{array}{l}\text { Key-ROESY } \\
\text { correlations }\end{array}$ \\
\hline Asp-1 & & & & \\
\hline 1 & 170.4 & & & \\
\hline 2 & 49.6 & 4.61 & & \\
\hline $2-\mathrm{NH}$ & & 8.12 & $2.49,2.62,4.61$ & $4.61,5.93$ \\
\hline 3 & 36.2 & $2.49,2.62$ & & \\
\hline 4 & 172.0 & & & \\
\hline Dap-2 & & & & \\
\hline 1 & 170.6 & & & \\
\hline 2 & 48.6 & 4.65 & $3.09,3.57,4.65$, & $3.57,4.61$ \\
\hline $2-\mathrm{NH}$ & & 8.23 & 7.49 & \\
\hline 3 & 39.9 & $3.09,3.57$ & & \\
\hline $3-\mathrm{NH}$ & & 7.49 & $3.09,3.57,4.65$, & $3.09,3.57,4.19$, \\
& & & & 4.65 \\
\hline Pip-3 & & & & \\
\hline 1 & 168.6 & & & \\
\hline 2 & 56.2 & 4.79 & $1.38,1.55,2.16$, & \\
\hline 3 & 26.6 & $1.55,2.17$ & & \\
\hline 4 & 20.4 & $1.38,1.52$ & & \\
\hline 5 & 24.7 & $1.22,1.57$ & & \\
\hline 6 & 39.9 & $2.86,4.33$ & & \\
\hline Gly-4 & & & & \\
\hline 1 & 169.6 & & & \\
\hline 2 & 41.9 & $3.62,3.96$ & & \\
\hline $2-\mathrm{NH}$ & & 8.07 & $3.62,3.96$ & $3.62,4.79$ \\
\hline Asp-5 & & & & \\
\hline 1 & 170.0 & & & \\
\hline 2 & 49.5 & 4.55 & & \\
\hline $2-\mathrm{NH}$ & & 8.26 & $2.53,2.74,4.55$ & $3.62,3.96,4.55$ \\
\hline 3 & 36.2 & $2.53,2.74$ & & \\
\hline 4 & 171.8 & & & \\
\hline Gly-6 & & & & \\
\hline 1 & 169.0 & & & \\
\hline 2 & 42.3 & 3.77 & & \\
\hline $2-\mathrm{NH}$ & & 8.11 & & \\
\hline Asp-7 & & & 3.77 & \\
\hline 1 & 171.1 & & & \\
\hline 2 & 50.3 & 4.48 & & \\
\hline $2-\mathrm{NH}$ & & 8.34 & & \\
\hline 3 & 36.1 & $2.55,2.70$ & & \\
\hline 4 & 171.8 & & & \\
\hline & & & & \\
\hline & & & \\
\hline
\end{tabular}

\begin{tabular}{|l|l|l|l|l|}
\hline Residue & $\begin{array}{l}{ }^{13} \mathrm{C} / \\
100 \mathrm{MHz}\end{array}$ & $\begin{array}{l}\mathrm{H} / 500 \mathrm{MHz} \\
\text { Mult }(\mathrm{J}, \mathrm{Hz})\end{array}$ & $\begin{array}{l}\text { Key-TOCSY } \\
\text { correlations }\end{array}$ & $\begin{array}{l}\text { Key-ROESY } \\
\text { correlations }\end{array}$ \\
\hline Gly-8 & & & & \\
\hline 1 & 169.4 & & & \\
\hline 2 & 42.2 & $3.69,3.79$ & & \\
\hline $2-\mathrm{NH}$ & & 7.92 & $3.69,3.79$ & $3.69,3.81,4.48$ \\
\hline Thr-9 & & & & \\
\hline 1 & 169.8 & & & \\
\hline 2 & 58.6 & 4.27 & & \\
\hline $2-\mathrm{NH}$ & & 7.82 & $1.02,3.81,4.27$ & $3.69,3.81,4.27$ \\
\hline 3 & 66.8 & 3.81 & & \\
\hline 4 & 19.6 & 1.02 & & \\
\hline Ile-10 & & & & \\
\hline 1 & 170.8 & & & \\
\hline 2 & 54.4 & 4.31 & & \\
\hline $2-\mathrm{NH}$ & & 7.67 & & \\
\hline 3 & 36.2 & 1.71 & $1.49,1.71,4.31$ & $4.27,4.31$ \\
\hline $3-\mathrm{Me}$ & 14.8 & 0.85 & & \\
\hline 4 & 24.3 & $1.05,1.49$ & & \\
\hline 5 & 10.5 & 0.75 & & \\
\hline Pro-11 & & & & \\
\hline 1 & 170.7 & & & \\
\hline 2 & 59.7 & 4.19 & & \\
\hline 3 & 29.4 & $1.71,2.00$ & & \\
\hline 4 & 24.6 & $1.79,1.91$ & & \\
\hline 5 & 47.2 & $3.50,3.76$ & & \\
\hline FA & & & & \\
\hline 1 & 165.1 & & & \\
\hline 2 & 124.1 & $5.93(\mathrm{~d}, 15.6)$ & $6.62,2.12,1.37$, & \\
\hline 3 & 143.3 & $6.62(\mathrm{dt}, 15.6)$ & & \\
\hline 4 & 31.4 & $2.12 \mathrm{~m}$ & & \\
\hline 5 & 27.9 & 1.37 & & \\
\hline 6 & 28.8 & 1.24 & & \\
\hline $7-10$ & $29.0-29.2$ & 1.23 & & \\
\hline 11 & 26.9 & 1.21 & & \\
\hline 12 & 38.6 & 1.10 & & \\
\hline 13 & 27.5 & 1.46 & & \\
\hline 14,15 & 22.5 & $0.81(\mathrm{~d}, 6.5)$ & $1.10,1.21,1.46$ & \\
\hline & & & & \\
\hline & & & \\
\hline & & & \\
\hline & & & \\
\hline & & & \\
\hline
\end{tabular}

structure of amphomycin ${ }^{3)}$, however, it was in contradiction with the sequence of fragment II. The $\alpha$-amino group of the Dap residue that forms a peptide linkage with the $\alpha$ carboxyl of the aspartyl residue in II is the same nitrogen atom bonding to the carboxyl group of Pip to form the pyrazine-1,4-dione ring in Id. Since peptide bonds cannot be generated under hydrolytic conditions, the only plausible explanation for all occurring fragments is that the peptide linkage between Dap and Asp in II was present originally, whereas the diketopiperazine moiety in Id was the result of a rearrangement. The ease of release of FA-Asp (IV) and the ready cleavage of the glycyl linkages during hydrolysis of 1 would allow the liberated carboxyl group of the pipecolic acid residue and the $\alpha$-amino group of Dap to react forming the ring closure in Id. It is well known that diketopiperazines can be formed by refluxing dipeptides in methanol ${ }^{4}$. A ROESY correlation observed for $\mathbf{1}$ between a glycine NH (Gly-4) and the $\alpha$-proton of Pip demonstrated that Pip was part of a larger cyclic structure. This was taken as additional evidence that the pyrazine-1,4-dione ring present in Ia or Id arose by condensation of a liberated peptide following initial cleavage of the Gly-Pip bond. The ${ }^{13} \mathrm{C}$ NMR data also failed to support the presence of a diketopiperazine in the intact antibiotic 1 , as it lacked a diagnostic upfield carbonyl resonance around $\delta 162$, characteristic of the diketopiperazine carbonyl of Pip in Ia. Although the aminosuccinimide present in III was recognized as an artifact created during the hydrolysis ${ }^{5)}$, the structure of III gave further support for the existence of the peptide linkage between the $\alpha$-amino group of Dap and the 
Fig. 2. Fragments formed in the partial acid hydrolysis of glycinocin A (1).

Ia:<smiles>NC[C@@H]1NC(=O)C2CCCCN2C1=O</smiles>

Ib: Thr-lle-Pro-Dap

Ic: Thr-lle-Pro

Id:<smiles>O=C1N[C@@H](CNO[PH3])C(=O)N2CCCCC12</smiles>

II: Thr-lle-Pro-Dap-Asp-FA

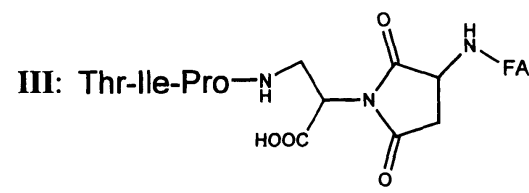

IV: Asp-FA

aspartyl residue of FA-Asp.

The remaining three glycine and two aspartic acid residues, suggested by the NMR data and required by the molecular formula, must be located between the threonine and pipecolic acid residues. MS-MS analysis showed a series of fragments at 1134, 976, 804, 632, 521, corresponding to initial cleavage between isoleucine and protonated proline, and then sequential losses of Ile, ThrGly, Asp-Gly, Asp-Gly, and Pip, respectively (Figure 3). ROESY correlations observed between two of the three glycyl NH protons $(\delta 7.92,8.11)$ and the $\alpha$-protons $(\delta$ $4.48,4.55)$ of the neighboring aspartyl residues, and between the third glycyl NH (Gly-4) at $\delta 8.07$ and the $\alpha$ proton of Pip at $\delta 4.79$ allowed completion of the cyclic peptide structure of glycinocin A (1) as shown.

\section{Absolute Stereochemistry of 1}

The absolute configurations of the amino acids were determined using Marfey's method. Hydrolysis of glycinocin (1) and its degradation products Ib, Id, and II yielded the corresponding amino acids. The acid hydrolysates were treated with Marfey's reagent, 5-fluoro2,4-dinitrophenyl- $L$-alanine amide (FDAA), the resulting FDAA derivatives were analyzed by reversed phase HPLC and LCMS. Each peak in the chromatographic traces was identified by comparing the retention time with that of the FDAA derivative of authentic amino acid. The Marfey's reagent derivatives of the amino acids liberated from 1 showed peaks matching $L$-Asp, $D$-allo-Thr, Gly, $L$-Pro, $D$ Pip, $L$-Ile, and $L$-Dap. Amino acid analysis of the fragments Ib, Id, and II gave consistent results. In all cases a small peak $(\sim 20 \%)$ corresponding to $D$-Dap was observed, owing to partial epimerization of $L$-Dap.

\section{Structures of $\mathbf{2}, \mathbf{3}$, and $\mathbf{4}$}

Glycinocin B (2) was isolated as an optically active off-white powder. Its molecular formula was established as $\mathrm{C}_{58} \mathrm{H}_{92} \mathrm{~N}_{12} \mathrm{O}_{19}$ by high resolution FT-ICR mass spectrometry. It only differed from the molecular formula of glycinocin $\mathrm{A}$ by the gain of a $\mathrm{CH}_{2}$ unit. The ${ }^{1} \mathrm{H}$ and ${ }^{13} \mathrm{C}$ NMR spectra of 2 were nearly identical to those recorded for 1 , indicating that the two compounds were closely related. MS/MS characterization of $\mathbf{2}$ revealed that it had the same amino acid residues and peptide sequence but a longer fatty chain. Partial acid hydrolysis of 2 led to isolation of the fatty acyl aspartic acid conjugate, homologous to IV, identified by MS and NMR data as $N$ (2-isohexadecenoyl) aspartic acid.

Glycinocin C (3) is an optically active metabolite whose molecular formula was determined to be $\mathrm{C}_{56} \mathrm{H}_{88} \mathrm{~N}_{12} \mathrm{O}_{19}$ by high resolution FT-ICR mass spectrometry, differing from the molecular formula of $\mathbf{1}$ by the lack of one $\mathrm{CH}_{2}$ unit. The MS-MS spectrum of $\mathbf{3}$ showed the identical fragmentation pattern as that of $\mathbf{1}$, revealing that it differed from $\mathbf{1}$ only in the fatty chain. Analysis of $\mathbf{3}$ using Marfey's method confirmed that it contained the same constituent amino acid residues. The nearly identical ${ }^{1} \mathrm{H}$ and ${ }^{13} \mathrm{C}$ NMR spectra of $\mathbf{3}$ to those recorded for 1 prompted the conclusion that compound 3 contained a 2 -isotetradecenoyl moiety. This was in a good agreement with its shorter retention time on reversed-phase HPLC.

The molecular formula of glycinocin D (4) was established by high resolution FT-ICR mass spectrometry as $\mathrm{C}_{56} \mathrm{H}_{88} \mathrm{~N}_{12} \mathrm{O}_{19}$, identical to compound 3, differing from the molecular formula of $\mathbf{1}$ by the loss of one carbon and two hydrogen atoms. Amino acid analysis of compound 4 using the Marfey's methodology demonstrated that it contained the same constituent amino.acid residues as those of 1 except that $L$-Val replaced $L$-Ile. MS/MS analysis identified the location for the valine residue as well as the sequence of 4 as shown in Figure 1. Comparison of the NMR data obtained for $\mathbf{4}$ with those of $\mathbf{1}$ revealed that it had the same fatty acid moiety as that of $\mathbf{1}$. 
Fig. 3. MS/MS fragmentation pattern of glycinocin (1).

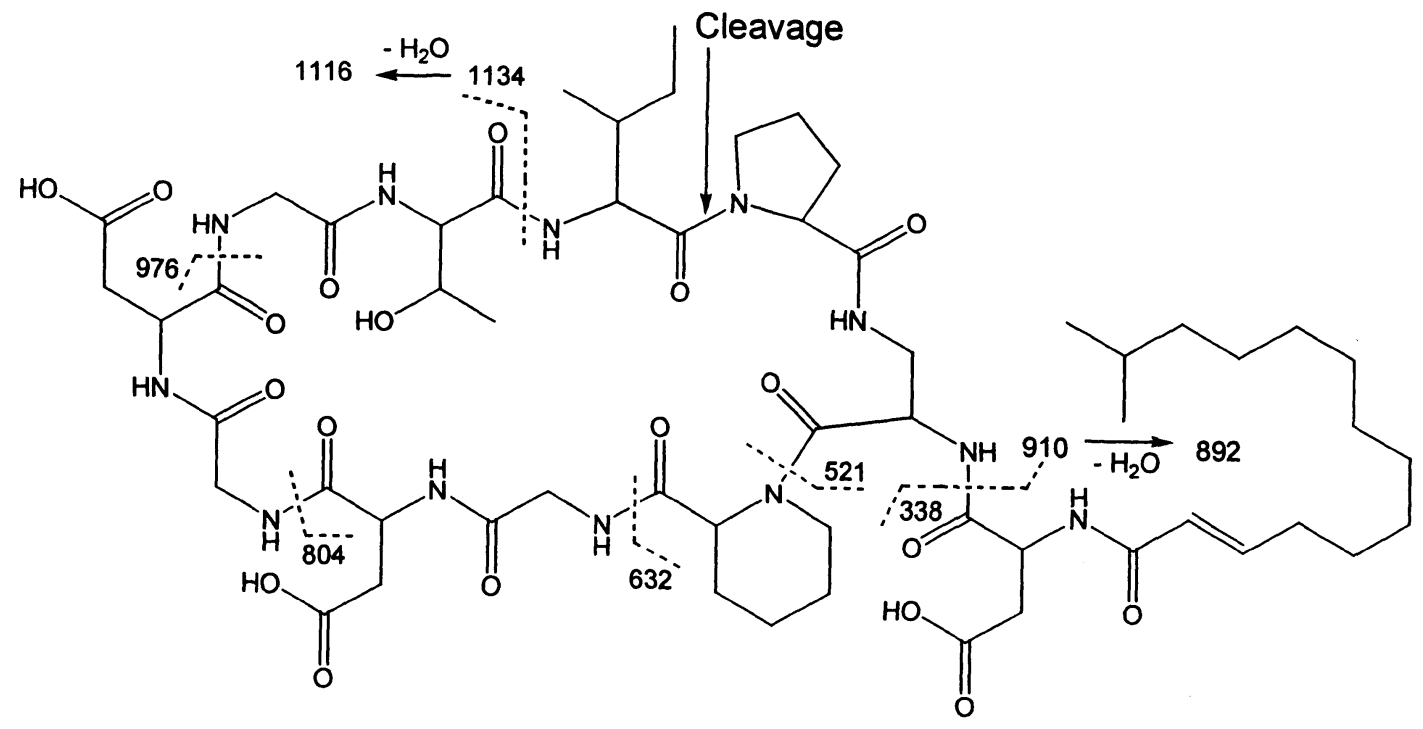

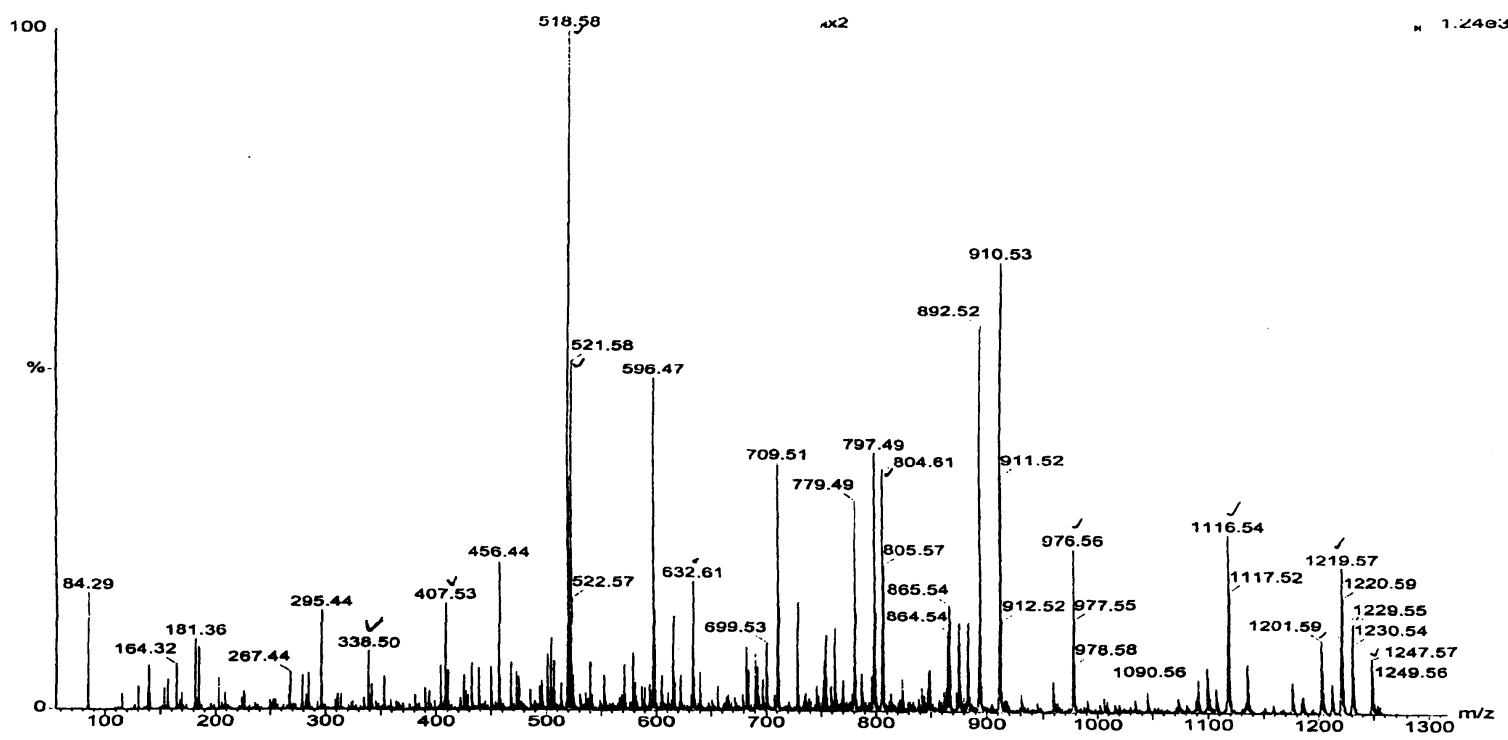

\section{Conclusion}

Glycinocins represent a new series of cyclic lipopeptide antibiotics related to amphomycin. The distinguishing features of this series compared to amphomycin are the substitution of $D$-allo-Thr for the Dab(9) unit and $L$-Dap for the branching residue $\operatorname{Dab}(2)$, the replacement of the Me-Asp(4) residue with Gly, and the shift of the unsaturation in the fatty acyl chain from $\Delta^{3,4}$ to $\Delta^{2,3}$ creating an $\alpha, \beta$-unsaturated amide. The facile condensation of the Dap and Pip residues forming the diketopiperazine moiety during hydrolytic experiments has resulted in numerous postulated structures containing the artifactual unit that should now be revisited and corrected. In a recent conclusive study VERTESY ${ }^{6}$ ) also pointed out that the linear structure proposed by BODANSZKY in 1973 for amphomycin (aka glumamycin) should be corrected to the original cyclic one reported in 1965 by FuJINo ${ }^{7)}$.

\section{Experimental}

\section{General}

Optical rotations were taken on a Jasco DIP-370 digital 
polarimeter. Electrospray mass spectra and MS/MS data were measured on a VG Quattro-I triple quadrupole mass spectrometer. High resolution mass spectra were recorded on a 9.4 Tesla FT-ICR mass spectrometer. NMR spectra were recorded on a Bruker DRX 500 or AMX 300 spectrometer in deuterated solvents. ${ }^{1} \mathrm{H}$ and ${ }^{13} \mathrm{C}$ chemical shifts were measured in parts per million relative to partially deuterated solvent peaks of DMSO- $d_{6}$ at $\delta 2.49$ and $\delta 39.5$ for ${ }^{1} \mathrm{H}$ and ${ }^{13} \mathrm{C}$ NMR signals, respectively. ${ }^{1} \mathrm{H}-{ }^{1} \mathrm{H}$ coupling constants were measured from 1D proton spectra and are given in Hertz. A typical NMR data set measured for a compound included carbon, DEPT, proton, COSY, TOCSY (40 ms mixing time), HMQC, HMBC, NOESY or ROESY, and HMQC-TOCSY spectra. All 2D experiments were run non-spinning.

\section{HPLC Systems}

A Hewlett-Packard 1100M LC system with diode array detection and a Finnigan LCQ LC-MS system employing a YMC-ODS-A reversed phase column $(5 \mu 4.6 \times 150 \mathrm{~mm}$ or $4.6 \times 250 \mathrm{~mm}$ ) were used for analysis of fractions, degradation products, and Marfey's derivatives. Preparative HPLC separations were accomplished on a MetaChem Inertsil ODS-3 (C18, 20×250 mm) column. Fractions from preparative columns were generally collected using ISCO Foxy fractional collector and monitored by analytical HPLC or LC-MS. All solvents used were obtained from EM Science or J. T. Baker, Inc., and were of the highest commercially available purity.

\section{Isolation}

One liter of the fermentation broth of AW998 culture was centrifuged. The aqueous layer was partitioned against $n$-BuOH $(500 \mathrm{ml} \times 2)$ and the mycelial mass was extracted with $\mathrm{MeOH}(100 \mathrm{ml} \times 2)$. Both $n-\mathrm{BuOH}$ and $\mathrm{MeOH}$ extracts were combined and concentrated under reduced pressure to dryness. The dried residue was redissolved in DMF$\mathrm{MeOH}-\mathrm{H}_{2} \mathrm{O}(2: 1: 1)$ and loaded onto a reversed phase HPLC column (MetaChem ODS-3, $20 \mathrm{~mm} \times 250 \mathrm{~mm}$ ). The column was washed with a gradient of $50 \sim 70 \%$ acetonitrile in $0.05 \%$ trifluoroacetic acid buffer over 60 minutes at a flow rate of $7 \mathrm{ml} /$ minute, and the peaks were detected by UV absorbance at $215 \mathrm{~nm}$. The antibiotics were eluted with the following retention times in minutes: 34 for glycinocin C, 38 for D, 46 for A, and 55 for B. The major components glycinocin A (70 mg) and B (15 mg) appeared reasonably pure as judged by ${ }^{1} \mathrm{H}$ NMR analysis. A secondary HPLC purification of the minor components $C$ and D using the same HPLC column with a gradient elution of $40 \sim 60 \%$ acetonitrile in $0.05 \%$ TFA buffer over 90 minutes yielded pure glycinocin C (9.3 mg, 61 minutes $)$ and D (9.5 mg, 68 minutes).

Glycinocin A (1): White powder; $[\alpha]_{\mathrm{D}}^{25}=-22^{\circ}(c 0.68$, $\mathrm{MeOH}$ ); HRMS (FT-ICR) measured 1246.6453, calculated 1246.6448 for $\mathrm{C}_{57} \mathrm{H}_{90} \mathrm{~N}_{12} \mathrm{O}_{19}$; LRESMS $\mathrm{m} / \mathrm{z} 1247.6$ $(\mathrm{M}+\mathrm{H})^{+}, 910.5(30 \%) ; \mathrm{MS} / \mathrm{MS}$ data see Figure $3 .{ }^{1} \mathrm{H}$ and ${ }^{13} \mathrm{C}$ NMR see Table 1.

Glycinocin B (2): White powder; $[\alpha]_{\mathrm{D}}^{25}=-19^{\circ}(c 0.41$, $\mathrm{MeOH}$ ); HRMS (FT-ICR) measured 1260.6610, calculated 1260.6605 for $\mathrm{C}_{58} \mathrm{H}_{92} \mathrm{~N}_{12} \mathrm{O}_{19}$; LRESMS $\mathrm{m} / \mathrm{z} \quad 1261.5$ $(\mathrm{M}+\mathrm{H})^{+}, \quad 910.4(35 \%)$; NMR spectra in DMSO- $d_{6}$ $(300 \mathrm{MHz}):{ }^{1} \mathrm{H},{ }^{13} \mathrm{C}$, g-COSY, TOCSY, g-HMQC, gHMBC, similar to $\mathbf{1}$, no detailed assignments. FA-Asp: LRESMS $m / z 370.2(\mathrm{M}+\mathrm{H})^{+}, 368.3(\mathrm{M}-\mathrm{H})^{-} ;{ }^{1} \mathrm{H}$ NMR $\left(300 \mathrm{MHz}, \mathrm{DMSO}-d_{6}\right) \delta$ Asp H-2, 4.55 (q, 7.0 Hz); NH, 8.15 (d, 7.8); H-3, 2.70 (dd, 5.6, 16.5); H-3', 2.57 (dd, 7.4, 16.5); FA H-2, 5.95 (d, 15.5); H-3, 6.61 (dt, 15.5, 6.9); H-4, 2.11 (q, 6.9); H-5, 1.39 (m); H-6 to H-12, 1.23; H-13, 1.12 (m); H-14, 1.48 (m); H-15/H-16, 0.83 (d, 6.6).

Glycinocin C (3): White powder; $[\alpha]_{\mathrm{D}}^{25}=-13^{\circ}(c 0.28$, $\mathrm{MeOH}$ ); HRMS (FT-ICR) measured 1232.6294, calculated 1232.6289 for $\mathrm{C}_{56} \mathrm{H}_{88} \mathrm{~N}_{12} \mathrm{O}_{19}$; LRESMS $\mathrm{m} / \mathrm{z} \quad 1233.4$ $(\mathrm{M}+\mathrm{H})^{+}, 910.4(50 \%) ;{ }^{1} \mathrm{H}$ NMR $\left(300 \mathrm{MHz}\right.$, DMSO- $\left.d_{6}\right)$ spectrum; Amino acid analysis (Marfey's method), same as 1.

Glycinocin D (4): White powder; $[\alpha]_{\mathrm{D}}^{25}=-18^{\circ}(c 0.33$, $\mathrm{MeOH}$ ); HRMS (FT-ICR) provided $\mathrm{m} / \mathrm{z} 1233.6356$ $(\mathrm{M}+\mathrm{H})^{+}$, calculated 1233.6362 for $\mathrm{C}_{56} \mathrm{H}_{89} \mathrm{~N}_{12} \mathrm{O}_{19}^{+}$; LRESMS $m / z 1233.4(\mathrm{M}+\mathrm{H})^{+}, 896.3(40 \%) ;{ }^{1} \mathrm{H}$ NMR and ROESY spectra $\left(300 \mathrm{MHz}\right.$, DMSO- $\left.d_{6}\right) ;$ Amino acid analysis: $L$-Asp $\times 3, L$-Dap, $D$-Pip, Gly $\times 3, D$-allo-Thr, $L$ Val (32.7 minutes), $L$-Pro; Standard: $L$-Val, 32.7 minutes; $D$-Val, 37.6 minutes; $L$-Ile, 36.8 minutes; $D$-Ile, 45.1 minutes; YMC-ODS-A column $(5 \mu, 4.6 \times 250 \mathrm{~mm})$.

\section{Determination of Absolute Stereochemistry}

Standards: The standard amino acid $(0.2 \mathrm{mg}$, Sigma) was dissolved in water $(30 \mu \mathrm{l})$, then treated with $1 \% 1$-fluoro2,4-dinitrophenyl-5- $L$-alanine amide (FDAA) in acetone $(60 \mu \mathrm{l})$ and $6 \%$ triethylamine in water $(30 \mu \mathrm{l})$ at $40^{\circ} \mathrm{C}$ for 1 hour. After cooling to room temperature, the derivative was analyzed by reversed-phase HPLC detected by UV absorption at $340 \mathrm{~nm}$. The column (YMC-ODS-A, $5 \mu$, $4.6 \times 150 \mathrm{~mm}$ ) was eluted with a linear gradient of (A) $\mathrm{CH}_{3} \mathrm{CN}$ and (B) $0.05 \%$ aqueous TFA from $10 \%$ to $40 \%$ (A) over 30 minutes followed by isocratic elution with $40 \%$ (A) at a flow rate of $1 \mathrm{ml} /$ minute. The standards gave the following retention time in minutes: 19.8 for FDAA; 18.4 for Gly; 16.8 for $L, 17.8$ for $D$-Asp; 29.8 for $L, 29.8 / 30.7$ for $L / D$-Dap; 27.1 for $L, 27.1 / 25.4$ for $L / D$-Pip; 16.4 for $L$, 
19.5 for $D$-Thr; 16.6 for $L, 17.6$ for $D$-allo-Thr; 28.9 for $L$, 33.3 for $D$-Ile; 20.5 for $L, 21.6$ for $D$-Pro.

Peptides: Glycinocin A (1, $1 \mathrm{mg})$ was dissolved in $6 \mathrm{~N}$ $\mathrm{HCl}(0.5 \mathrm{ml})$ and heated at $108^{\circ} \mathrm{C}$ in a sealed Pyrex vial overnight to yield the corresponding amino acids. The cooled reaction mixture was evaporated to dryness under reduced pressure and the trace of $\mathrm{HCl}$ was removed from the residual acid hydrolysate by repeated evaporation from frozen water $(0.5 \mathrm{ml})$. The amino acid mixture was then treated as the standards above [1\% FDAA $(150 \mu \mathrm{l})$ and $\left.6 \% \mathrm{Et}_{3} \mathrm{~N}(80 \mu \mathrm{l})\right]$. The mixture of FDAA derivatives was filtered, the filtrate was diluted with water $(150 \mu \mathrm{l})$ and analyzed by HPLC. The FDAA derivatives of the amino acids liberated from 1 showed peaks at 16.8, 17.7, 18.2, $19.8,20.5,25.4,28.8$, and 29.8 minutes, matching the retention time of $L$-Asp, $D$-allo-Thr, Gly, FDAA, $L$-Pro, $D$ Pip, $L$-Ile, and $L$-Dap.

\section{Partial Acid Hydrolysis}

Glycinocin A (20 mg) was dissolved in $3 \mathrm{~N} \mathrm{HCl}$ solution $\left(\mathrm{MeOH}-\mathrm{H}_{2} \mathrm{O}, 100 \mathrm{ml}\right)$ and heated at $80^{\circ} \mathrm{C}$ for 5 hours. The reaction mixture was concentrated to about $3 \mathrm{ml}$ and chromatographed by a preparative HPLC (MetaChem ODS-3, $20 \times 250 \mathrm{~mm}$ ). The column was eluted with a linear gradient of $50 \%$ to $80 \% \mathrm{MeCN}$ and $0.1 \%$ TFA buffer over 50 minutes at $7 \mathrm{ml} /$ minute. Peaks eluted in sequence were $\mathbf{I}$ ( 8 minutes), II (23 minutes), III (32 minutes), IV (80 minutes). Fraction I was further purified using the same HPLC column with a linear gradient of $5 \%$ to $25 \% \mathrm{MeCN}$ and $0.1 \%$ TFA buffer over 60 minutes to yield Ia (50 minutes), Ib (63 minutes), Ic (75 minutes), and Id (78 minutes). A huge front peak eluted at 8 minutes was not collected. Methyl ester derivatives of Ib and III were also isolated. Some fragments were further purified by a second round of HPLC.

Ia: Colorless glass; LRESMS $m / z 198.1(\mathrm{M}+\mathrm{H})^{+} ;{ }^{1} \mathrm{H}$ NMR (300 MHz, DMSO- $d_{6}$ ) $\delta$ Dap H-2, $4.26(\mathrm{t}, 5.6)$; NH2, 8.17 (bs); H-3, 3.14 (bs); $\mathrm{NH}_{2}-3,7.93$ (bs); Pip H-2, 3.85 (dd, 12.0, 2.1); H-3, 1.48 (m), 2.07 (m); H-4, 1.51 (m), 1.84 (m); H-5, 1.28 (m), 1.64 (m); H-6, 2.54 (td, 11.5, 2.2), 4.42 (dd, 1.7, 13.0); ${ }^{13} \mathrm{C}$ NMR (75 MHz, DMSO- $d_{6}$ ) $\delta$ Dap C-1, 166.3; C-2, 51.0; C-3, 40.6; Pip C-1, 162.2; C-2, 58.1; C-3, 29.9; C-4, 23.2; C-5, 23.8; C-6, 41.6.

Ib: Colorless glass; LRESMS $m / z 416.1(\mathrm{M}+\mathrm{H})^{+} ;{ }^{1} \mathrm{H}$ NMR (300 MHz, DMSO- $\left.d_{6}\right) \delta$ Thr H-2, 3.80; NH, 7.99 (bs); H-3, 4.10 (quin, 5.5); H-4, 1.04 (d, 6.4); Ile H-2, 4.36; $\mathrm{NH}, 8.72$ (d, 8.2); H-3, 1.80; $\mathrm{CH}_{3}-3,0.90$ (d, 6.7); H-4, 1.12, 1.48; H-5, 0.82 (t, 7.2); Pro H-2, 4.26; H-3, 1.85, 1.98; H-4, 1.85, 1.96; H-5, 3.61, 3.83; Dap H-2, 3.91 (bs); $\mathrm{NH}-2,8.23$ (bs); H-3, 3.36, 3.69; NH-3, 8.31 (t, 5.6); ${ }^{13} \mathrm{C}$
NMR (HMQC, DMSO- $d_{6}$ ) $\delta$ Thr C-2, 57.5; C-3, 65.0; C4, 17.5; Ile C-2, 54.5; C-3, 36.0; $\mathrm{CH}_{3}-3,14.5$; C-4, 24.0; C5, 10.5; Pro C-2, 59.5, C-3, 29.0, C-4, 24.0; C-5, 46.5; Dap C-2, 53.0; C-3, 39.0; Amino acid analysis: $D$-allo-Thr, $L$ Pro, $L$-Ile, $L$-Dap.

Ic: Colorless glass; LRESMS $m / z 330.0(\mathrm{M}+\mathrm{H})^{+} ;{ }^{1} \mathrm{H}$ NMR (300 MHz, DMSO- $d_{6}$ ) $\delta$ Thr H-2, 3.80; NH, 8.00 (bs); H-3, 4.11 (quin, 5.5); H-4, 1.05 (d, 6.5); Ile H-2, 4.38 (t, 8.7); NH, 8.77 (d, 8.4); H-3, 1.80; $\mathrm{CH}_{3}-3,0.93$ (d, 6.8); $\mathrm{H}-4,1.12$, 1.50; H-5, 0.81 (t, 7.3); Pro H-2, 4.21; H-3, 1.88, 1.95; H-4, 1.90, 2.12; H-5, 3.62, 3.84.

Id: Colorless glasses; LRESMS $m / z 509.1(\mathrm{M}+\mathrm{H})^{+} ;{ }^{1} \mathrm{H}$ NMR (300 MHz, DMSO- $d_{6}$ ) $\delta$ Thr H-2, 3.77; NH, 8.00 (bs); H-3, 4.12; H-4, 1.06 (d, 6.4); Ile H-2, 4.36; NH, 8.77; $\mathrm{H}-3,1.82 ; \mathrm{CH}_{3}-3,0.93 ; \mathrm{H}-4,1.13,1.51 ; \mathrm{H}-5,0.82$ (t, 7.2); Pro H-2, 4.26; H-3, 1.88, 1.96; H-4, 1.90, 2.12; H-5, 3.62, 3.81; Dap H-2, 3.91 (bs); NH-2, 7.77 (d, 1.6); H-3, 3.37, 3.54; NH-3, 8.01; Pip H-2, 4.41; H-3, 1.27, 2.50; H-4, 1.40, 1.61 ; H-5, 1.32, 1.82; H-6, 2.08, 3.83; Amino acid analysis: $D$-allo-Thr, $L$-Pro, $D$-Pip, $L$-Ile, $L$-Dap.

II: Colorless glass; LRESMS m/z $753.3(\mathrm{M}+\mathrm{H})^{+}, 652.4$ (4\%), 539.4 (35\%), $442.3(10 \%) ; 338.3$ (3\%); ${ }^{1} \mathrm{H}$ NMR and TOCSY spectra $\left(300 \mathrm{MHz}\right.$, DMSO- $\left.d_{6}\right)$; Amino acid analysis: $L$-Asp, $D$-allo-Thr, $L$-Pro, $L$-Ile, $L$-Dap.

III: Colorless glass; LRESMS $m / z 735.3(\mathrm{M}+\mathrm{H})^{+}, 634.3$ (10\%), 521.4 (50\%), 424.2 (20\%); ${ }^{1} \mathrm{H}$ NMR (300 MHz, DMSO-d $\left.{ }_{6}\right) \delta$ Thr H-2, 3.82; NH, 7.98; H-3, 4.12; OH-3, 3.33; $\mathrm{H}-4,1.05$; Ile $\mathrm{H}-2,4.38 ; \mathrm{NH}, 8.69 ; \mathrm{H}-3,1.80 ; \mathrm{CH}_{3}-3$, 0.93; H-4, 1.11, 1.47; H-5, 0.81; Pro H-2, 4.16; H-3, 1.79, 1.91 ; H-4, 1.69, 1.91; H-5, 3.59, 3.78; Dap H-2, 4.62; H-3, 3.44, 3.83; NH-3, 7.84; Asp H-2, 4.76; NH, 8.72; H-3, 2.59, 3.00; FA H-2, 5.89; H-3, 6.66; H-4, 2.13; H-5, 1.40; H-6 to H-11, 1.23; H-12, 1.12; H-13, 1.48; H-14/H-15, 0.83 (d, 6.6).

IV: Colorless glass; LRESMS $\mathrm{m} / \mathrm{z} 356.1(\mathrm{M}+\mathrm{H})^{+} ;{ }^{1} \mathrm{H}$ NMR (300 MHz, DMSO- $d_{6}$ ) $\delta$ Asp H-2, 4.59 (q, 7.0 Hz); NH, 8.19 (d, 8.0); H-3, 2.69 (dd, 5.6, 16.2); H-3', 2.58 (dd, 7.4, 16.2); FA H-2, 5.94 (d, 15.4); H-3, 6.61 (dt, 15.4, 6.9); $\mathrm{H}-4,2.12$ (q, 6.8); H-5, 1.38 (m); H-6 to H-11, 1.23; H-12, 1.12 (m); H-13, 1.48 (m); H-14/H-15, 0.83 (d, 6.6).

\section{Acknowledgments}

The authors would like to thank our colleagues Mr. J. D. KORSHALLA and S. LUCKMAN for providing the fermentations, Dr. D. M. Roll and Mrs. J. K. MANNING for the initial isolation work, Drs. K. JANOTA, P. D. SCHNIER, and J. S. AshCROFT for MS and NMR studies, Mr. A. SCHORK for OR data collection. The authors also wish to thank Dr. G. SCHLINGMANN for insightful discussions. 


\section{References}

1) He, H.; W. D. Ding, V. S. Bernan, A. D. Richardson, C. M. Ireland, M. Greenstein, G. A. Ellestad \& G. T. CARTER: Lomaiviticins $\mathrm{A}$ and $\mathrm{B}$, potent antitumor antibiotics from Micromonospora Lomaivitiensis. J. Am. Chem. Soc. 123: 5362 5363, 2001

Kong, F.; N. Zhao, M. M. Siegel, K. Janota, J. S. AshCROFT, F. E. KoEHN, D. B. BORders \& G. T. CARTER: Saccharomicins, novel heptadecaglycoside antibiotics effective against multidrug-resistant bacteria. J. Am. Chem. Soc. 120: 13301 13311, 1998

2) Wise, Jr. E. M.: Peptides, In Kirk-Othmer Encycl. Chem. Technol., Kroschwitz, J. I. \& Howe-Grant, M., 4th edn., John Wiley \& Sons, New York, 3: 266 305, 1992

3) BodAnszky, M.; G. F. Sigler \& A. BodAnsky: Structure of the peptide antibiotic amphomycin. J. Am. Chem. Soc. 95: $2352 \sim 2357,1973$
4) Masuda, Y.; M. Tanihara, Y. Imanishi \& T. Higashima: Hydrolysis of active esters of aliphatic carboxylic acids with cyclic dipeptide catalysts consisting of $L$-histidine and different aliphatic $\alpha$-amino acids. Bull. Chem. Soc. Jpn. 58: 497 504, 1985

5) Ondetti, M. A.; A. Deer, J. T. Sheehan, J. Plescec \& O. KoCY: Side reaction in the synthesis of peptides containing the aspartylglycyl sequence. Biochemistry 7 : 4069 4075, 1968

6) Vertesy, L.; E. Ehlers, H. Kogler, M. Kurz, J. Meiwes, G. Seibert, M. Vogel \& P. Hammann: Friulimicins: novel lipopeptide antibiotics with peptidoglycan synthesis inhibiting activity from Actinoplanes friuliensis sp. nov., II. Isolation and structural characterization. J. Antibiotics 53: 816 827, 2000

7) FuJiNo, M.: Glumamycin, a new antibiotic. VI. Approach to the amino acid and sequence. Bull. Chem. Soc. Jap. 38: $517 \sim 522,1965$ 